\section{Measles outbreak investigation in a highly vaccinated commu- nity in the Centre region of Cameroon}

\author{
Haddison C. Eposi, ${ }^{1}$ Ngwafor A. \\ Randolph, ${ }^{2}$ Kagina M. Benjamin ${ }^{3}$ \\ ${ }^{1}$ Saa District Health Service, Centre \\ Regional Delegation of Public Health, \\ Cameroon; ${ }^{2}$ Institute for Global Health, \\ University College London, United \\ Kingdom; ${ }^{3}$ Vaccines For Africa \\ Initiative (VACFA), University of Cape \\ Town, South Africa
}

\begin{abstract}
Background: Measles remains a threat in many African settings due to sub-optimal routine immunisation and catchup campaigns. The Global Vaccine Action Plan goal to eliminate measles by 2020 remains unmet as several countries reported an increase in cases in 2019. In Cameroon, a measles-rubella vaccination campaign was organised in 2019 to reduce the cohort of susceptible children. However, in 2020, eleven suspected cases of measles were notified in the Sa'a Health District and five were confirmed.
\end{abstract}

Objective: This report summarizes a measles outbreak investigation and contact tracing in a highly vaccinated community residing in the Sa'a Health District of Cameroon.

Methods: Outbreak investigations were carried out in the Sa'a, Nlong-Onambele and Nkolmgbana health areas from 18 to 21 February 2020. A register review from December 2019 to February 2020 was carried out in all health facilities of the affected health areas. followed by contact tracing in the community.

Results: Thirty households were visited in four neighbourhoods. Six missed Epidemiologically-linked cases were discovered in the community, bringing the total number of suspected and confirmed cases to 17. Thirty-five percent of the cases had not received any measles-containing vaccine; $35 \%$ of the cases were aged 5 years or older; $53 \%$ had history of travel. Community transmission only occurred in the Sa'a health area through a breakthrough case.

Conclusions: This outbreak investigation portrayed the role that adequate vaccination coverage plays in preventing widespread outbreaks. Nonetheless, community sensitisation and routine immunisation require strengthening in order to erase pockets of susceptible children.

\section{Introduction}

The global effort to fight measles and rubella have been guided by key recommendations and subsequent implementations of the 2012-2020 Global Strategic Plan for measles and rubella eradication, some of which are: effective surveillance; achieve and attain high levels of population immunity; outbreak preparedness and response. ${ }^{1}$ However, the target set by the Global Vaccine Action Plan to eliminate measles in the World Health OrganisationAfrican (WHO-AFRO) region by 2020 remains unmet. ${ }^{2}$ In fact, according to the $\mathrm{WHO}$, the AFRO region recorded a $700 \%$ increase in the number of measles cases in $2019 .{ }^{3}$ Cameroon is one of the countries in Africa that was reported to have a measles outbreak in 2019, with a total of 2729 cases, an $800 \%$ increase from $2018 .{ }^{4}$ One of the reasons for outbreaks of VaccinePreventable Diseases (VPDs) including measles, is sub-optimal vaccination coverages during routine immunisation. Therefore, measles catch up and mass campaigns are recommended in order to attain optimal population immunity and to mitigate the occurrence of outbreaks.

In Cameroon, like many other countries, measles vaccine is provided to children through routine immunisation services delivered by the Expanded Programme on Immunisation (EPI). The EPI in Cameroon targets 0-15 months old children. The measles immunisation programme began in Cameroon in 1965 and by 1974 the vaccine was administered only to 9-month-old children as a single dose. ${ }^{5}$ Since 2015 the vaccine is administered as a combined Measles-Rubella (MR) vaccine with first dose at 9 months of age. The national coverage for the first dose of the MR vaccine in 2019 was reported to be $71 \%{ }^{4}$ There were variations in coverage of the first dose for different regions. For example, three regions, including the Centre region recorded a coverage above the national target of $83 \% .{ }^{4}$ The second dose of the MR vaccine targeting 15-months-old children was introduced into the EPI in 2020. As expected, uptake of the second dose in the Centre region remained very low after only 3 months of implementation. Uptake of the second dose is expected to improve with time as well as with mass vaccination campaigns.

Since 2002, Cameroon has carried out 5 follow up measles vaccination campaigns, with the last one taking place in 2015 . Reports show that measles epidemics in the country peak every three years. ${ }^{5}$ The last
Correspondence: Haddison C. Eposi, Saa District Health Service, Centre Regional Delegation of Public Health, Cameroon.

Tel.: +237 699385785

E-mail: eposihaddison@yahoo.com

Key words: Measles; outbreak; investigation; vaccination; Cameroon.

Acknowledgements: Dr Tambasho Afizu, Central Technical Group-Expanded Programme on Immunisation, Ministry of Health, Cameroon; Mr Enyegue Mbia Guy, Sa'a District Health Service, Centre RDPH, Cameroon; Mr Okala Jean Didier, Nlong Onambele Integrated Health Centre, Sa'a DHS, Cameroon; Mme Ndzana Dior, Ayissile Integrated Health Centre, Sa'a DHS, Cameroon

Contributions: HEC conceived the study and collected the data. HEC and NAR analysed the data and wrote the manuscript with supervision from KMB. All authors read and approved the final manuscript

Conflict of interest: The authors have no conflict of interest to declare.

Availability of data and materials: All data generated or analysed during this study are included in this published article

Ethics approval and consent to participate: The findings of this study were derived from routine immunisation activities so no ethical approval was required.

Informed consent: Written informed consent was obtained from a legally authorized representative(s) for anonymized patient information to be published in this article.

Received for publication: 11 December 2020 Revision received: 23 March 2021.

Accepted for publication: 23 March 2021

This work is licensed under a Creative Commons Attribution NonCommercial 4.0 License (CC BY-NC 4.0).

(C) Copyright: the Author(s),2021

Licensee PAGEPress, Italy

Journal of Public Health in Africa 2021: 12:1775 doi:10.4081/jphia.2021.1775

peak was in 2015 thus the next follow up vaccination campaign was scheduled for 2018 in order to prevent an eventual peak. Unfortunately, the 2018 campaign was rescheduled to 2019. We speculate the rescheduling resulted to the resurgence in measles cases across the country due to an increase in the number of susceptible children. ${ }^{4}$ In effect, by the end of 2019, 47 of the 189 health districts in the country had notified 3 confirmed cases within 28 con- 
secutive days, thus crossing the epidemic threshold. ${ }^{4}$ According to national guidelines, a response vaccination coverage targeting children 9-59 months is recommended in any locality where an epidemic occurs. It was therefore imperative that the measles campaign initially scheduled for 2018 be carried out, to reduce the number of susceptible children to prevent further outbreaks.

It is in this context that the 2019 national MR campaign coupled with Vitamin A supplementation and deworming targeting children aged 9 to 59 months was carried out in the Sa'a Health District (HD). The Sa'a HD is one of the 30 HDs of the Centre region of Cameroon. The MR campaign coordination was done at the District level with supervision from the Regional Technical Group-Expanded Programme on Immunisation. The co-ordination was similar to that of the Polio campaign done in 2018 as previously described by Haddison et $a l .{ }^{6}$ The MR vaccine (Serum Institute, India) in vials of 10 doses was used for the campaign. The campaign took place from the 4th to the 10th of December 2019. Only the fixed post strategy in health facilities and temporary fixed post strategy in areas such as markets, churches and motor parks were used since this was an injectable vaccine. A total of 9164 out of 9308 children were vaccinated, resulting to an administrative campaign coverage of $97 \%$.

To ascertain how successful the campaign was, a MR campaign coverage survey in the district was evaluated using WHO's cluster immunisation methodology. ${ }^{7}$ Three health areas-Sa'a, Nlong-Onambele and Nkolmgbana were purposively chosen, with twenty-four clusters randomly chosen by balloting. Vaccination was ascertained either by visual inspection of the vaccination card distributed during the campaign or by recall. Out of the 479 children surveyed, 463 were vaccinated giving a MR campaign coverage of $96 \%$.

Between the first and sixth epidemiological week of 2020, eleven suspected cases of measles were notified in the Sa'a HD where a successful MR campaign had been ascertained. Serum specimens from the suspected cases were sent to Centre Pasteur du Cameroun for analysis. Of the eleven, five specimens tested positive, surpassing the threshold for a measles epidemic. Therefore, sampling of suspected cases was suspended according to national policies pending an outbreak investigation and response. It is worth noting that there were zero confirmed measles cases in the district out of the 11 suspected cases notified in 2018 and 13 cases notified in 2019. Surprisingly, measles outbreaks were declared in 15 of the 30 health districts of the Centre region within the same period. We report a measles outbreak investigation and contact tracing in a highly vaccinated community residing in Nlong-Onambele, Sa'a, and Nkolmgbana health areas in the Sa'a HD.

\section{Materials and Methods}

\section{Ethical considerations}

The findings of this study were derived from routine immunisation activities so no ethical approval was required.

\section{Setting}

Investigations were carried out in three health areas; i) Sa'a HA which is the largest HA of the district and most populous with a population of 29307 inhabitants. This HA has the highest concentration of health facilities in the district including the district hospital; ii) Nlong-Onambele HA with a population of 6230 inhabitants served by one health facility; iii) and Nkolmgbana $\mathrm{HA}$, the smallest HA of the district with a population of 2274 inhabitants served by one health facility. This HA shares borders with Sa'a HA.

\section{Investigation process}

Investigations were carried out in two phases from 18 to 21 February 2020 (epidemiological week 8). Firstly, a register review from December 2019 to February 2020 was carried out in all health facilities of the affected HAs. Secondly, contact tracing was carried out in the community. The homes of the positive cases were visited first, then homes within a $100 \mathrm{~m}$ radius of the homes of the positive cases and any suspected case found in the community were visited.

\section{Case definitions}

Suspected case: Anyone with fever and maculo-papular rash plus either cough, coryza or conjunctivitis. ${ }^{8}$

Confirmed case: Any suspected case with laboratory positive results for $\operatorname{IgM}$ antibody. ${ }^{8}$

Missed case: Any suspected case who was not notified but later identified in the community.

Nä̈ve case: Any case who had never been vaccinated with a measles containing vaccine (MCV).

Breakthrough case: Any case who had received a dose of $\mathrm{MCV}$.

\section{Data collection and analysis}

Data collected included socio-demo- graphic characteristics of the cases, date of onset of the symptoms, date of consultation, vaccination and travel history as well as contact with other suspected cases. Data were subsequently entered and analysed using Microsoft Excel 2016 (Microsoft Corp., Redmond, Washington, United States). Proportions were calculated based on the total number of cases (confirmed, missed, suspected).

\section{Results}

A total of 30 households were visited in four neighbourhoods including the homes of the five positive cases. Majority of the cases from all the health facilities that were recorded in the register review were identified. Six missed epidemiologically linked cases were discovered in the community thus bringing the total number of suspected and confirmed cases to 17 from epidemiological week 1 to 8 (Figure 1).

\section{Clinical presentation}

The ages of the cases ranged from 10 months to 42 years (Table 1). All the cases presented with a fever and rash and were considered as having mild to moderate disease except for one case who developed rectorrhagia thus considered as severe disease (Table 2). No deaths were recorded.

\section{Profiling of the cases}

There was a similar proportion of breakthrough and naïve infections. Vaccination status could not be ascertained in $29.4 \%$ of the cases. Only one child between the ages of 9 months to 5 years had received two doses of the MR vaccine. Most of the cases $(52.9 \%)$ had recently travelled out of the district (Table 1).

\section{Investigation and contact tracing}

Investigations began in the Ebogo neighbourhood in Saa HA where the confirmed case had been notified then moved to Nlong Onambele and Nkolmbagna health areas. At the end of the investigations, the following facts were reconstituted based on the onset of symptoms in each health area.

During epidemiological week 1, the index case, a resident of Djoungolo HD (80km from Sa'a HD) visited her cousin, patient 2 who resided in the Bami neighbourhood in Saa HA. During the visit the index case developed symptoms of measles, which led to a cascade of transmissions in Saa HA from epidemiological week 1 to 5 (Figure 2). During epidemiological week 3, patient 2 developed symptoms of measles and was hospitalised in Ebogo where he also schooled. Investigators found out that 
patients 3 and 4 (a missed case) who developed symptoms during epidemiological week 5 were his classmates. No other pupils were reported sick by the school principal. Back in the Bami neighbourhood investigators discovered patients 5 and 6 who were neighbours to patient 2 .

Though patient 7 and his father (patient 8) had both consulted for measles in Ebogo during epidemiological week 5 , investigators found no epidemiological link between them and patients 1-6. Investigators learnt that patients 7 and 8 , residents of Djoungolo HD had come to Sa'a for the burial of a family member who allegedly died of measles in Djoungolo HD. Upon arrival they already had symptoms of measles. Investigators were unable to locate patients 9 and 10 who had been notified by the health centre.

In the Nlong Onambele HA, investigators found out that both cases notified by the health facility, patient 11 and patient 12 , residents of Djoungolo HD had arrived Sa'a sick during epidemiological weeks 1 and 2 respectively in search of traditional medicine. No other cases were found in the community. Also, no epidemiological link could be found between them and the other cases in the district.
In the Nkolmgbana HA, during epidemiological week 3 patient 13 developed mild symptoms (fever) that were not classified as measles related until a week later. Investigators learnt patient 13 had visited

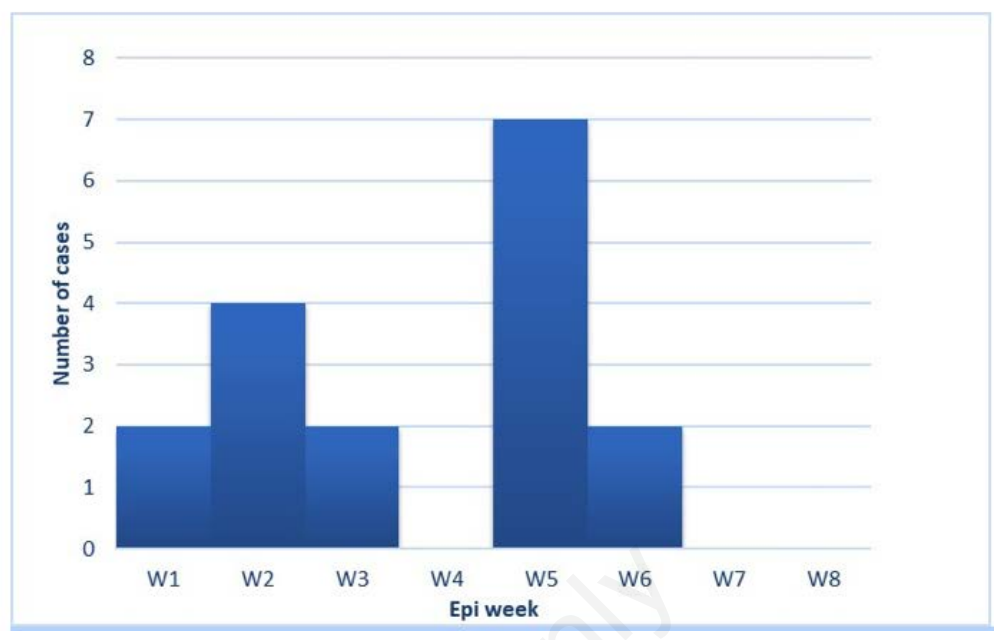

Figure 1. Epidemiological curve for measles cases from epidemiological week 1 to 8 by date of onset of symptoms.

Table 1. Characteristics of the measles cases.

\begin{tabular}{|c|c|c|c|c|c|c|c|c|c|}
\hline Patient & Age & Notified & $\begin{array}{l}\text { IgM } \\
\text { sera }\end{array}$ & $\begin{array}{c}\text { Onset of symptoms } \\
\text { (epidemiological } \\
\text { week) }\end{array}$ & $\begin{array}{l}\text { Doses } \\
\text { of MR }\end{array}$ & $\begin{array}{c}\text { History of } \\
\text { travel }\end{array}$ & $\begin{array}{l}\text { Contact with } \\
\text { another case }\end{array}$ & $\begin{array}{c}\text { Health area of } \\
\text { notification } \\
\text { in Sa'a HD }\end{array}$ & $\begin{array}{l}\text { Health } \\
\text { district of } \\
\text { residence }\end{array}$ \\
\hline 1 & $1.5 y$ & No & ND & 1 & 0 & Yes & Yes & Sa'a & Djoungolo \\
\hline 2 & $5 y$ & Yes & Pos & 3 & 2 & No & Yes & Sa'a & Sa'a \\
\hline 3 & $8 y$ & Yes & Pos & 5 & 0 & No & Yes & Sa'a & Sa'a \\
\hline 4 & $6 y$ & No & ND & 5 & 1 & No & Yes & Sa'a & Sa'a \\
\hline 5 & $4 y$ & Yes & Neg & 5 & 1 & No & Yes & Sa'a & Sa'a \\
\hline 6 & $1.5 y$ & Yes & Neg & 5 & 0 & No & Yes & Sa'a & Sa'a \\
\hline 7 & $2 y$ & Yes & Pos & 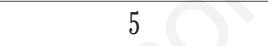 & Unknown & Yes & Yes & Sa'a & Djoungolo \\
\hline 8 & $42 y$ & Yes & Neg & 5 & Unknown & Yes & Yes & Sa'a & Djoungolo \\
\hline 9 & $14 \mathrm{mths}$ & No & ND & 5 & 0 & No & Unknown & Sa'a & Sa’a \\
\hline 10 & $5 y$ & Yes & Neg & 6 & 1 & No & Unknown & Sa'a & Sa'a \\
\hline 11 & $1.5 \mathrm{y}$ & Yes & Neg & 1 & Unknown & Yes & No & Nlong Onambele & Djoungolo \\
\hline 12 & $20 y$ & Yes & Pos & 2 & Unknown & Yes & No & Nlong Onambele & Djoungolo \\
\hline 13 & $10 \mathrm{mths}$ & Yes & Pos & 3 & 1 & Yes & No & Nkolmbgana & Sa'a \\
\hline 14 & ly & No & ND & 2 & 1 & No & Yes & Nkolmbgana & Sa'a \\
\hline 15 & $3 y$ & No & ND & 2 & 0 & Yes & Yes & Nkolmbgana & Sa'a \\
\hline 16 & ly & No & ND & 2 & 0 & Yes & Yes & Nkolmbgana & Sa'a \\
\hline 17 & $5 y$ & Yes & $\mathrm{Neg}$ & 6 & 1 & Yes & No & Lepopomo & Sa'a \\
\hline
\end{tabular}

ND: Not done, Pos: Positive, Neg: Negative, y: years, mths: months.

Table 2. Distribution of symptoms among cases.

\begin{tabular}{lccc} 
Symptoms & Naiive cases & Breakthrough cases & Indeterminate cases \\
$\mathbf{N}=6(35.3 \%)$ & $\mathbf{N}=6(35.3 \%)$ & 5 \\
$(29.4 \%)$ & 5 \\
Fever & 6 & 6 & 5 \\
Rash & 6 & 3 & 1 \\
\hline Cough & 3 & 3 & 1 \\
Conjunctivitis & 2 & 3 & 1 \\
\hline Coryza & 4 & 1 & 0 \\
Rectorrhagia & 0 & 6 & 1 \\
\hline
\end{tabular}


Djoungolo HD prior to the onset of the symptoms. In the same community, three other missed cases were discovered: patients 14, 15 and 16. All three lived in the same house and developed symptoms of measles during epidemiological week 2 . Even though patients 13, 14, 15 and 16 lived in the same neighbourhood there was no evidence of contact between patient 13's household and that of patients 14, 15 and 16.

Based on these findings it was concluded that: i) the cases identified in the different health areas were not linked, but rather were isolated events; ii) the virus was imported into Sa'a HD most probably from Djoungolo HD which was known to have an ongoing measles outbreak and; iii) no reactive vaccination campaign was needed due to the high vaccination coverage of the December 2019 MR measles campaign and the limited spread of the outbreak.

\section{Discussion}

We report a measles outbreak investigation and contact tracing in a community that on record, had been highly vaccinated with the MR vaccine through a mass campaign. Our key findings were: i) thirty-five $(35 \%)$ percent of the cases had not received any MR vaccine; ii) thirty-five percent (35\%) of the cases were 5 years or older; iii) more than half $(53 \%)$ of the cases had history of travel; and, iv) community transmission only occurred in Sa'a health area through a breakthrough case.

Measles Containing Vaccine (MCV) has been proven to provide long term protection against the measles virus hence its recommendation into EPI schedules by WHO. ${ }^{9}$ Despite measles vaccines being in the Cameroonian vaccination schedule for many years, and regular follow-up measles vaccination campaign, we still discovered children who had not received any MCV. Many of the unvaccinated children had parents who were considered as being negligent since they had older siblings who had been vaccinated. Awareness of the importance of vaccines remains a key influencer to vaccine uptake. ${ }^{10}$ Parents with poor knowledge on the immunisation calendar or the duration of protection of vaccines are less likely to seek care. ${ }^{10,11}$

During the investigations, parents of patient 8 appeared to be vaccine hesitant. Even when faced with the measles infection of their child the parents still refused to have the other siblings vaccinated. In some settings, vaccine hesitancy has been reported to arise due to loss of confidence in vaccines among other reasons. ${ }^{12}$ In our settings, we propose urgent research to identify the determinants of vaccine hesitancy as well as development of optimal interventions to counteract the determinants. Vaccine hesitancy can lead to outbreaks of VPDs as a result of pockets of susceptible children. ${ }^{12}$

A high number of breakthrough cases (41\%) were observed during the outbreak. Vaccine failure has been reported as the main reason why vaccinated children develop measles. ${ }^{13}$ Primary vaccine failure occurs mostly in children who receive MCV1 before the age of 12 months. ${ }^{13,14}$ Despite this, WHO still recommends MCV1 vaccination at 9 months in areas endemic for measles in order to protect susceptible infants. ${ }^{9}$ In Cameroon, MCV1 is administered at 9 months, so, vaccine failure may explain why children who had been vaccinated presented with symptoms. A universal second dose of MCV is recommended to provide immunity for children who may have had a primary vaccine failure. ${ }^{9}$ The MR vaccination campaign would have served as the perfect opportunity for this $2^{\text {nd }}$ dose, unfortunately almost all the breakthrough cases did not receive the vaccine. Moreover, a third of the cases were out of the target group for the campaign so could not have benefited from this additional dose.

Contrary to what is expected, we had adults in our setting who were infected with the measles virus. Unfortunately, their vaccination status was unknown. Based on their ages, there were neither eligible for the national MR campaign in 2015 nor 2019. Several studies report that measles immunity acquired naturally or by vaccination may be lifelong. ${ }^{15,16}$ Some authors have argued that most of these studies were carried out during a period when there was natural boosting from the wild measles virus which is no longer the case with elimination of
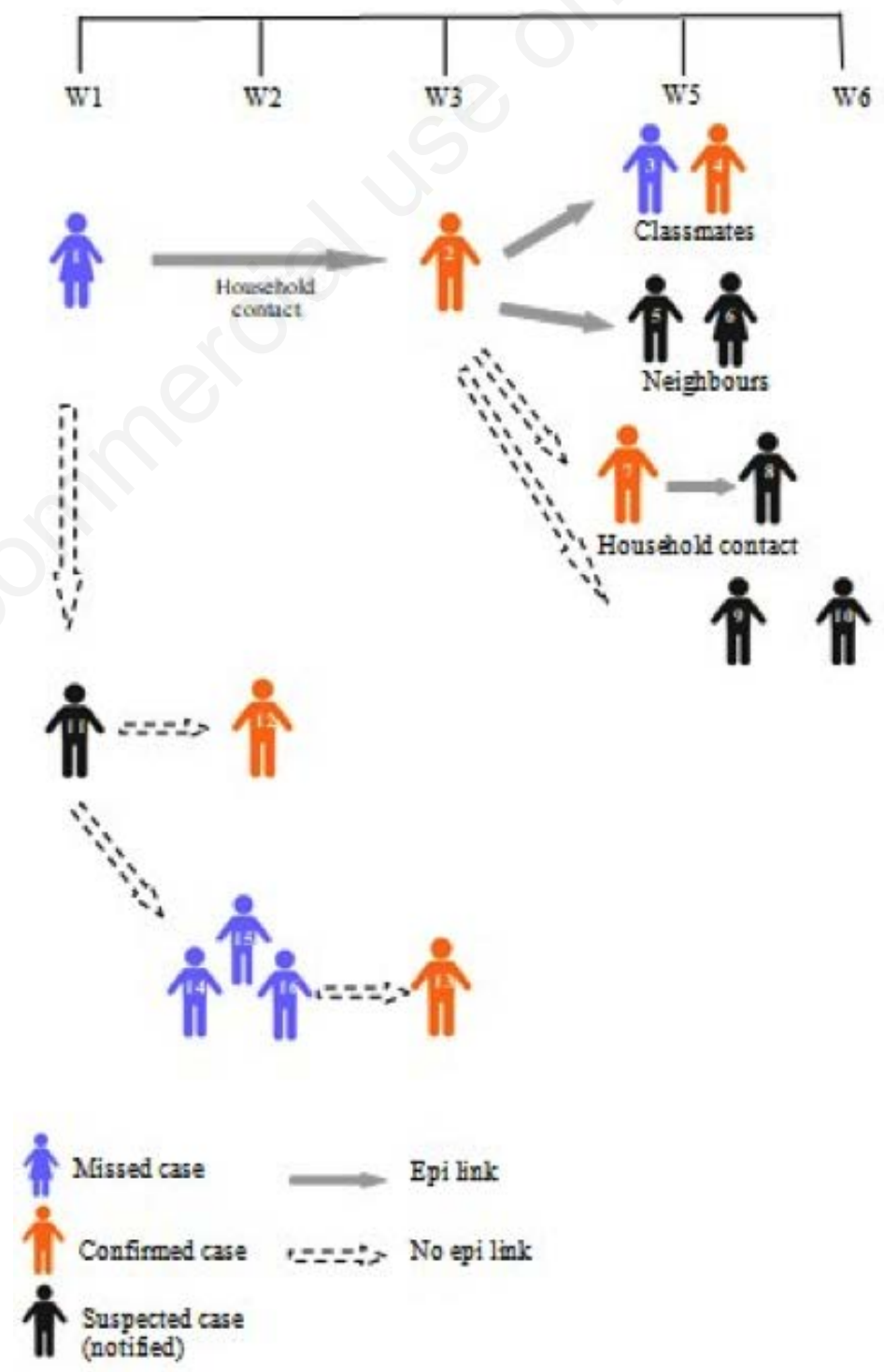

Figure 2. Transmission chain in Sa'a health district. 
measles in most settings. ${ }^{17,18}$ In Cameroon, measles is still endemic so in the event that these adults were vaccinated they would have benefited from this natural boosting. We can only speculate that they were naïve cases and there may be several more in the community.

During the period that the measles outbreak was declared in the Sa'a HD, other neighbouring urban HDs like Djoungolo had already declared measles epidemics. Many of the positive cases declared in Sa'a HD were residents of these districts or had recently travelled from these districts and arrived Sa'a while already infected. In many African settings, patients tend to leave the cities to rural areas in search of traditional or alternative treatment. ${ }^{19}$ This favours the importation of diseases which may lead to outbreaks in susceptible populations. This was the case in our setting and we assume the outbreak was very localised due to herd immunity.

Studies reporting outbreaks in vaccinated populations rarely describe onward transmission from breakthrough cases. ${ }^{20-22}$ However, we observed this phenomenon in our setting. Patient 2 who had received 2 doses of the MR vaccine was the only contact to his classmates and neighbours in Sa'a health area. Moreover, he developed a severe form of the disease which is not in accordance with reports which describe mild disease in breakthrough cases. ${ }^{20-22}$ Nonetheless, a similar case was described by Rosen et al., in the United States. ${ }^{23}$

\section{Limitations}

This outbreak investigation had several limitations. Firstly, cases were considered as breakthrough cases based on vaccination history alone instead of IgG serology. No $\mathrm{IgG}$ serology could be requested as the reference lab only conducts IgM serology for measles diagnosis. Also, vaccination history was based on recall, which may be biased. Secondly, since the community was highly vaccinated, some cases may have been missed if they presented with milder symptoms. Nonetheless, we believe there was no other community transmission because no cases were declared from epidemiological week 7. Lastly, this investigation was only carried out in three health areas so careful generalisation of the results is needed.

\section{Conclusions}

This outbreak investigation portrayed the role adequate vaccination coverage plays in preventing widespread outbreaks. Nonetheless, community sensitisation and routine immunisation requires strengthen- ing in order to erase pockets of susceptible children.

\section{References}

1. WHO. Global Measles and Rubella strategic plan 2012-2020. Accessed 04 June 2020. Available from: https://www.who.int/immunization/diseases/measles/global_coordination/en/i ndex $3 . h t m l$

2. WHO. Global Vaccine Action Plan 2011-2020. Accessed 04 June 2020. Available form: https://www.who.int/immunization/glo bal_vaccine_action_plan/GVAP_doc_2 011 2020/en

3. World Health Organisation. New measles surveillance data for 2019. Accessed 04 June 2020. Available from: https://www.who.int/immunization/new sroom/measles-data-2019/en/

4. Ministry Of Health. Expanded programme on immunisation annual report, 2019. Cameroon: Ministry Of Health.

5. Cummings DAT, Moss WJ, Long K, et al. Improved measles surveillance in Cameroon reveals two major dynamic patterns of incidence. Int $\mathrm{J}$ Infect Dis 2006;10:148-55.

6. Haddison EC, Ngono D, Kouamen GT, Kagina BM. Successful polio supplementary immunisation activities in a security compromised zone Experiences from the Southwest region of Cameroon. Vaccine 2018;36:6961-7.

7. World Health Organization Vaccination Coverage Cluster Surveys: Reference Manual. 2018 Accessed 09 January 2020. Available from: https://www. who.int/immunization/documents/who ivb 18.09/en/

8. World Health Organisation.WHO-recommended surveillance standard of measles. Accessed 10 June 2020. Available from: https://www. who.int/immunization/monitoring_surveillance/burden/vpd/surveillance_type /active/measles standards/en/

9. World Health Organisation. Measles vaccine: WHO position paper-April 2017. Weekly Epidemiolog Record 2017;92:205-28.

10. Smith LE, Amlôt R, Weinman J, et al. A systematic review of factors affecting vaccine uptake in young children. Vaccine 2017;35:6059-69.

11. Vonasek BJ, Bajunirwe F, Jacobson LE, et al. Do maternal knowledge and attitudes towards childhood immunizations in rural Uganda correlate with complete childhood vaccination? PloS One 2016;11:e0150131.
12. Salmon DA, Dudley MZ, Glanz JM, Omer SB. vaccine hesitancy: causes, consequences, and a call to action. Am J Prev Med 2015;49:S391-8.

13. Ashbaugh HR, Cherry JD, Hoff NA, et al. Measles antibody levels among vaccinated and unvaccinated children 659 months of age in the Democratic Republic of the Congo, 2013-2014. Vaccine 2020;38:2258-65.

14. Carazo S, Billard MN, Boutin A, De Serres G. Effect of age at vaccination on the measles vaccine effectiveness and immunogenicity: systematic review and meta-analysis. BMC Infect Dis 2020;20:251.

15. Dine MS, Hutchins SS, Thomas A, et al. Persistence of vaccine-induced antibody to measles 26-33 years after vaccination. J Infect Dis 2004;189:S123-30.

16. Ramsay ME, Moffatt D, O'Connor M. Measles vaccine: a 27-year follow-up. Epidemiol Infect 1994;112:409-12.

17. Whittle HC, Aaby P, Samb B, et al. Effect of subclinical infection on maintaining immunity against measles in vaccinated children in West Africa. Lancet 1999;353:98-102.

18. Paunio M, Hedman K, Davidkin I, et al. Secondary measles vaccine failures identified by measurement of IgG avidity: high occurrence among teenagers vaccinated at a young age. Epidemiol Infect 2000;124:263-71.

19. James PB, Wardle J, Steel A, Adams J. Traditional, complementary and alternative medicine use in Sub-Saharan Africa: a systematic review. BMJ Glob Health 2018;3:e000895.

20. Sundell N, Dotevall L, Sansone M, Andersson M, Lindh M, Wahlberg T, et al. Measles outbreak in Gothenburg urban area, Sweden, 2017 to 2018: low viral load in breakthrough infections. Euro Surveill 2019;24:1900114.

21. Avramovich E, Indenbaum V, Haber M, et al. Measles outbreak in a highly vaccinated population - Israel, July-August 2017. MMWR Morb Mortal Wkly Rep 2018;67:1186-8.

22. Berry L, Palmer T, Wells F, et al. Nosocomial outbreak of measles amongst a highly vaccinated population in an English hospital setting. Infect Prev Practice 2019;1:100018.

23. Rosen JB, Rota JS, Hickman CJ, et al. Outbreak of measles among persons with prior evidence of immunity, New York City, 2011. Cli Infect Dis 2014;58:1205-10. 